\title{
The multi-weighted type fractional fourier transform scheme and its application over wireless communications
}

\author{
Yong $\mathrm{Li}^{1,2,3^{*}}$, Zhiqun Song ${ }^{1,2}$ and Xuejun Sha ${ }^{3}$
}

\begin{abstract}
Researching on the relationships among different multiple weighted type fractional Fourier transform (multi-WFRFT) schemes, we in this paper provide the modulation order relation of different multi-WFRFT in theory. Moreover, we also prove that the two matrix spaces (i.e., 4-WFRFT and multi-WFRFT) are equivalent. To apply multi-WFRFT into the wireless communication, we design the implementation structure of multi-WFRFT (in particular, for $2^{n}$-WFRFT scheme). Furthermore, the generalized hybrid carrier modulation (GHCM) system has been proposed in this paper, which can be degenerated to the classical hybrid carrier modulation (HCM) system with special parameters. We also verify the modulation order relationship between multi-WFRFT by numerical simulations. Leveraging the theory and structure of multi-WFRFT, we finally discuss its potential applications over secure communication and multiple input multiple output (MIMO) system.
\end{abstract}

Keywords: Multi-WFRFT, Wireless communication, MIMO system, Secure communication

\section{Introduction}

Recently, 4-weighted type fractional Fourier transform (4-WFRFT) has been popular in channel equalization, narrow-band interference (NBI) suppression, and signal processing [1-6]. Moreover, 4-WFRFT-based hybrid carrier modulation (HCM) system, due to its special structure, can achieve better performance than the traditional single carrier modulation (SCM) system and orthogonal frequency division multiplexing (OFDM) system under doubly selective (DS) channels $[1,3,6]$. The reason is that OFDM system will be plagued by inter-carrier interference (ICI) due to the highly time variance under DS channels, and the effectiveness of SCM system is impaired due to inter-symbol interference (ISI) cause by large timedelay spread. Meanwhile, HCM system, convergence of SCM and OFDM signal, can make the signal and residual interference evenly distribute in the time-frequency plane. In this case, it is less possible to produce the

*Correspondence: young_li_54@126.com

'Science and Technology on Communication Networks Laboratory, Shijiazhuang, China

${ }^{2}$ The 54th Research Institute of China Electronics Technology Group Corporation, Shijiazhuang, China

Full list of author information is available at the end of the article strong interference and performs better under DS channels. Although, the complexity of HCM tolerably increase by $\mathcal{O}(K \log (K))$ comparing with OFDM system.

However, there are little reports to research on multiple WFRFT (multi-WFRFT), which can be as the generalized formation of 4-WFRFT on wireless communications. Furthermore, the modulation order relationship between different multi-WFRFT schemes is also vague in theory. There are many different constitutions for multiWFRFT according to [5], such as the classical fractional Fourier transform (CFRFT)-based multi-WFRFT [7-11]. However, the standard WFRFT (i.e., 4-WFRFT)-based multi-WFRFT is interesting due to its implement structure. Ran et al. in [5] have provided the explanation of multi-WFRFT in mathematics. Unfortunately, the implementation structure of multi-WFRFT and explanation in physical are still ambiguous, which will obstruct its application on wireless communications.

To apply multi-WFRFT to wireless communications, we in this paper prove the relationship of modulation orders between multi-WFRFT and 4-WFRFT in theory, and can be described a simple formula, i.e., $\alpha_{M}=\frac{M}{4} \alpha_{4}$ when multi-WFRFT and 4-WFRFT of the same signal are equal $\left(\alpha_{M}\right.$ and $\alpha_{4}$ are the modulation orders for multi-WFRFT 
and 4-WFRFT, respectively). As a corollary, we also prove the relationship of modulation orders among multiWFRFT with different $M$. Furthermore, some important properties of multi-WFRFT have also been given in this paper. We finally prove that any $N \times N$ matrix spaces for multi-WFRFT are equivalent.

We also provide the implementation structure of multiWFRFT (in special, $2^{n}$-WFRFT). Furthermore, the generalized hybrid carrier modulation (GHCM) system has been proposed in this paper, which can be degenerated to the classical hybrid carrier modulation (HCM) system with special parameters. We also verify the modulation order relationship between multi-WFRFT by numerical simulations. Leveraging the theory and implementation structure of multi-WFRFT, we finally analyze the potential applications on wireless communications.

The rest of this paper is organized as follows. The basic knowledge of WFRFT is introduced in Section 2. Furthermore, the modulation order relationships between different multi-WFRFT will be proved in Section 3. To apply multi-WFRFT system into wireless communication, we provide the implementation structure for multi-WFRFT system (in special, $2^{n}$-WFRFT system) and its complexity requirements in Section 4. We then propose a GHCM system and provide some numerical simulations in Section 5. The potential applications of multi-WFRFT is also discussed in Section 5. We conclude the whole paper in Section 6.

Notations: $\alpha_{m}$ is the modulation order for $m$-WFRFT $(m \geq 4)$. And $\mathbf{W}_{m}^{\alpha_{m}}$ denotes the $m$-WFRFT matrix. Then, $A_{l}\left(\alpha_{4}\right)(l=0 \sim 3)$ is the weight coefficient of 4WFRFT while $B_{m, l}\left(\alpha_{m}\right)(l=0 \sim m-1)$ is the weight coefficient of m-WFRFT $(m>4)$. F is the normalized discrete Fourier transform, and the elements of $\mathbf{F}$, $[\mathbf{F}]_{j, k}=1 / \sqrt{N} \exp (-2 \pi i j k / N), j, k=0,1, \ldots, N-1$. Moreover, $\langle.\rangle_{N}$ denotes the modulo- $N$ calculation. At last, $\delta($. is the Kronecker delta. In this paper, M-WFRFT is used in the processing of proof; meanwhile, $\mathrm{m}$-WFRFT is used in other places.

\section{The basic knowledge for WFRFT}

The $\alpha_{4}$-order 4 -WFRFT of the original signal $X$ with length of $N$ can be defined as follows:

$$
\mathfrak{F}^{\alpha_{4}} X=\mathbf{W}_{4}^{\alpha_{4}} X
$$

where $\mathfrak{F}^{\alpha_{4}}$ is the 4-WFRFT operator. $\alpha_{4}$ terms the modulation order and can be any real number. $\mathbf{W}_{4}^{\alpha_{4}}$ is the $N \times N$ 4-WFRFT matrix with

$$
\mathbf{W}_{4}^{\alpha_{4}}=\sum_{l=0}^{3} A_{l}\left(\alpha_{4}\right) \mathbf{F}^{l},
$$

in which, the subscript 4 denotes 4-WFRFT. $A_{l}\left(\alpha_{4}\right)(l=$ $0 \sim 3, l \in \mathbb{Z}$ ) is the weight coefficients of 4-WFRFT, can be expressed as [12]:

$$
A_{l}\left(\alpha_{4}\right)=\frac{1}{4} \frac{1-\exp \left[-2 i \pi\left(\alpha_{4}-l\right)\right]}{1-\exp \left[-2 i \pi\left(\alpha_{4}-l\right) / 4\right]} .
$$

$\mathbf{F}$ is the discrete Fourier transform (DFT) matrix. Accordingly, $\mathbf{F}^{l}$ is the $l$-time Fourier transform $[5,12]$. Note that, $\mathbf{F}^{4}=\mathbf{F}^{0}=\mathbf{I}$ due to the 4-periodicity of Fourier transform [5]. Also, the 4-WFRFT simplifies to Fourier transform (FT) when $\alpha_{4}=1$. Moreover, the identity operation can be obtained when $\alpha_{4}=0$. For any real $\alpha_{4}$ and $\beta_{4}$, the following additive characteristic holds $[1,3]$ :

$$
\mathbf{W}_{4}^{\alpha_{4}+\beta_{4}}=\mathbf{W}_{4}^{\alpha_{4}} \mathbf{W}_{4}^{\beta_{4}}=\mathbf{W}_{4}^{\beta_{4}} \mathbf{W}_{4}^{\alpha_{4}}
$$

There are various methods for defining multi-WFRFT [5]. Based upon 4-WFRFT, multi-WFRFT ( $M$-WFRFT, $M>4$ ) of the original signal $X$, can be defined as [5]:

$$
\mathfrak{F}_{M}^{\alpha_{M}} X=\mathbf{W}_{M}^{\alpha_{M}} X, M>4,
$$

with

$$
\mathbf{W}_{M}^{\alpha_{M}}=\sum_{l=0}^{M-1} B_{l}\left(\alpha_{M}\right) \mathbf{W}_{4}^{\left(\frac{4 l}{M}\right)}, M>4 .
$$

where $\mathbf{W}_{M}^{\alpha_{M}}$ denotes the $N \times N M$-WFRFT matrix with subscript of $M$. The definition of $\mathbf{W}_{M}^{\alpha_{M}}$, as shown in (6), will be employed in this paper unless otherwise noted.

To distinguish the weight coefficients between 4WFRFT and $M$-WFRFT, we employ $B_{l}\left(\alpha_{M}\right)(M>4)$ to represent the weight coefficient of $M$-WFRFT and can be written as:

$$
B_{l}\left(\alpha_{M}\right)=\frac{1}{M} \frac{1-\exp \left[-2 i \pi\left(\alpha_{M}-l\right)\right]}{1-\exp \left[-2 i \pi\left(\alpha_{M}-l\right) / M\right]}, M>4 .
$$

According to (3) and (7), the following relationships hold:

$$
A_{l}\left(\alpha_{4}\right)=A_{k}\left(\alpha_{4}-l+k\right), l, k=0 \sim 3, l, k \in \mathbb{Z},
$$

and

$$
B_{h}\left(\alpha_{M}\right)=B_{j}\left(\alpha_{M}-h+j\right), h, j=0 \sim(M-1), h, j \in \mathbb{Z} .
$$

We have derived the relationships of $A_{l}\left(\alpha_{4}\right)$ and $A_{k}\left(\alpha_{4}\right)$ via (8), and the relationships of $B_{h}\left(\alpha_{M}\right)$ and $B_{j}\left(\alpha_{M}\right)$ via (9). However, the order relationships between 4-WFRFT and 
M-WFRFT are vague. We will reveal these relationships in the next section.

\section{The modulation order relationships between 4-WFRFT and multi-WFRFT}

In this section, we will reveal the modulation order relationship between 4-WFRFT and multi-WFRFT, which is essential for realizing the nature characteristics of WFRFT.

Theorem 1 Assuming $\alpha_{4}$ and $\alpha_{M}$ are the modulation orders of 4-WFRFT and multi-WFRFT, respectively, and $\mathbf{W}_{4}^{\alpha_{4}}$ and $\mathbf{W}_{M}^{\alpha_{M}}$ denote the matrices for 4-WFRFT and multi-WFRFT, accordingly. If $\mathbf{W}_{4}^{\alpha_{4}}=\mathbf{W}_{M}^{\alpha_{M}}$, then

$$
\alpha_{M}=\frac{M}{4} \alpha_{4}, M>4,
$$

Proof Mathematical induction will be exploited in the proof process, which incorporates three steps.

- Step 1 We firstly prove that when $\mathbf{W}_{5}^{\alpha_{5}}=\mathbf{W}_{4}^{\alpha_{4}},(10)$ holds, i.e., $\alpha_{5}=\frac{5}{4} \alpha_{4}$.

Inserting $M=5$ into (6), then

$$
\mathbf{W}_{5}^{\alpha_{5}}=\sum_{p=0}^{4} B_{p}\left(\alpha_{5}\right) \mathbf{W}_{4}^{\frac{4 p}{5}}
$$

Note that $\mathbf{W}_{4}^{\frac{4 p}{5}}(p=0 \sim 4, p \in \mathbb{Z})$ involves the sum of four terms and can be expressed as:

$$
\mathbf{W}_{4}^{\frac{4 p}{5}}=\sum_{j=0}^{3} A_{j}\left(\frac{4 p}{5}\right) \mathbf{F}^{j}, p=0 \sim 4
$$

By substituting (12) into (11):

$$
\mathbf{W}_{5}^{\alpha_{5}}=\sum_{j=0}^{3} \Theta_{j}(\mathbf{A}, \mathbf{B}) \mathbf{F}^{j}
$$

where

$$
\Theta_{j}(\mathbf{A}, \mathbf{B})=\sum_{p=0}^{4} B_{p}\left(\alpha_{5}\right) A_{j}\left(\frac{4 p}{5}\right), j=0 \sim 3 .
$$

Comparing (2) with (13), $\mathbf{W}_{5}^{\alpha_{5}}=\mathbf{W}_{4}^{\alpha_{4}}$ holds if and only if each coefficient of $\mathbf{F}^{j}(j=0 \sim 3, j \in \mathbb{Z})$ is equal. For brevity, we employ the coefficient of $\mathbf{F}^{0}$ in (2) and (13) to obtain the modulation order relationship. When $j=0$, (14) can be expressed:

$$
\Theta_{0}(\mathbf{A}, \mathbf{B})=\sum_{p=0}^{4} \Lambda_{p},
$$

with

$$
\left\{\begin{array}{l}
\Lambda_{0}=\frac{1}{5} \frac{1-\exp \left(-2 \pi i \alpha_{5}\right)}{1-\exp \left(\frac{-2 \pi i \alpha_{5}}{5}\right)} \\
\Lambda_{1}=-\frac{1}{20} \frac{1-\exp \left(-2 \pi i \alpha_{5}\right)}{\exp \left(-\frac{2 \pi i}{5}\right)-\exp \left(\frac{-2 \pi i \alpha_{5}}{5}\right)} \\
\Lambda_{2}=\frac{1}{20} \frac{1-\exp \left(-2 \pi i \alpha_{5}\right)}{\exp \left(\frac{\pi i}{5}\right)+\exp \left(\frac{-2 \pi i \alpha_{5}}{5}\right)} \\
\Lambda_{3}=\frac{1}{20} \frac{1-\exp \left(-2 \pi i \alpha_{5}\right)}{\exp \left(-\frac{\pi i}{5}\right)+\exp \left(\frac{-2 \pi i \alpha_{5}}{5}\right)} \\
\Lambda_{4}=-\frac{1}{20} \frac{1-\exp \left(-2 \pi i \alpha_{5}\right)}{\exp \left(\frac{2 \pi i}{5}\right)-\exp \left(\frac{-2 \pi i \alpha_{5}}{5}\right)}
\end{array}\right.
$$

Through (16), we can derive that

$$
\sum_{p=0}^{4} \Lambda_{p}=\frac{U \sum_{j=0}^{3}(j+1) \exp \left(-2 j \pi i \alpha_{5} / 5\right)}{20 \sum_{p=0}^{4} \exp \left(-2 p \pi i \alpha_{5} / 5\right)}
$$

where $U=1-\exp \left(-2 \pi i \alpha_{5}\right)$.

Obviously, both numerator and denominator are geometric sequence or its expansion [13]. Therefore, (17) can be simplified:

$$
\sum_{p=1}^{4} \Lambda_{p}=\frac{1-5 \exp \left(-8 \pi i \alpha_{5} / 5\right)+4 \exp \left(-2 \pi i \alpha_{5} / 5\right)}{20\left[1-\exp \left(-2 \pi i \alpha_{5} / 5\right)\right]}
$$

Finally, according to (15) and (18), $\Theta_{0}(\mathbf{A}, \mathbf{B})$ can be expressed as follows:

$$
\Theta_{0}(\mathbf{A}, \mathbf{B})=\frac{1-\exp \left(-8 \pi i \alpha_{5} / 5\right)}{4\left[1-\exp \left(-2 \pi i \alpha_{5} / 5\right)\right]}
$$

To explicitly compare the coefficients between 4-WFRFT and M-WFRFT, we provide the expression of $A_{0}\left(\alpha_{4}\right)$ :

$$
A_{0}\left(\alpha_{4}\right)=\frac{1-\exp \left(-2 \pi i \alpha_{4}\right)}{4\left[1-\exp \left(-2 \pi i \alpha_{4} / 4\right)\right]}
$$

Exploiting $\Theta_{0}(\mathbf{A}, \mathbf{B})=A_{0}\left(\alpha_{4}\right)$, (19) and (20), we obtain that

$$
\alpha_{5}=\frac{5}{4} \alpha_{4}
$$

Until now, we have completed the proof of step 1.

- Step 2 Assuming that when $M=k(k \geq 5)$, Theorem 1 holds [14]. That is, if $\mathbf{W}_{k}^{\alpha_{k}}=\mathbf{W}_{4}^{\alpha_{4}}$, the following formula holds: 


$$
\alpha_{k}=\frac{k}{4} \alpha_{4}
$$

Then, we will employ (22) to prove Theorem 1 for $M=k+1$.

- Step 3 As stated previously, we will prove when $M=k+1,(10)$ holds. To distinguish the coefficients of $k$-WFRFT and $(k+1)$-WFRFT, we in this step employ $B_{k, l}$ and $B_{k+1, l}$ denote the 1 th weight coefficient for $k$-WFRFT and $(k+1)$-WFRFT, respectively.

We first derive the relationship between $\alpha_{k}$ and $\alpha_{k+1}$ when $\mathbf{W}_{k}^{\alpha_{k}}=\mathbf{W}_{k+1}^{\alpha_{k+1}} \cdot \mathbf{W}_{k+1}^{\alpha_{k+1}}$ can be written as:

$$
\mathbf{W}_{k+1}^{\alpha_{k+1}}=\sum_{q=0}^{k} B_{k, q}\left(\alpha_{k+1}\right) \mathbf{W}_{4}^{\frac{4 q}{k+1}}
$$

By substituting (22) into (23), we obtain

$$
\mathbf{W}_{k+1}^{\alpha_{k+1}}=\sum_{q=0}^{k} B_{k, q}\left(\alpha_{k+1}\right) \mathbf{W}_{k}^{k q}
$$

Based upon k-WFRFT, we can get the coefficients of $\left(\mathbf{F}^{\frac{4}{k}}\right)^{0}$ for both $\mathbf{W}_{k}^{\alpha_{k}}$ and $\mathbf{W}_{k+1}^{\alpha_{k+1}}$.

$$
\begin{aligned}
B_{k, 0}\left(\alpha_{k}\right) & =\frac{1-\exp \left(-2 \pi i \alpha_{k}\right)}{k\left[1-\exp \left(-2 \pi i \alpha_{k} / k\right)\right]} \\
\Lambda_{0}\left(\mathbf{B}_{k}, \mathbf{B}_{k+1}\right) & =\sum_{q=0}^{k} \Gamma_{q}, \\
\Gamma_{q} & =B_{k+1, q}\left(\alpha_{k+1}\right) B_{k, 0}\left(\frac{k q}{k+1}\right), \\
q & =0 \sim k, q \in \mathbb{Z} .
\end{aligned}
$$

with

$$
\Gamma_{q}=B_{k+1, q}\left(\alpha_{k+1}\right) B_{k, 0}\left(\frac{k q}{k+1}\right), q=0 \sim k, q \in \mathbb{Z}
$$

With observation and complex computation, $\sum_{q=1}^{k} \Gamma_{q}$ can be obtained:

$$
\sum_{q=1}^{k} \Gamma_{q}=\frac{V \sum_{j=0}^{k-1}(j+1) \exp \left(\frac{-2 j \pi i \alpha_{k+1}}{k+1}\right)}{k(k+1) \sum_{q=0}^{k} \exp \left(\frac{-2 q \pi i \alpha_{k+1}}{k+1}\right)}
$$

with

$$
V=1-\exp \left(-2 \pi i \alpha_{k+1}\right)
$$

In this case, (29) can be expressed as:

$$
\sum_{q=1}^{k} \Gamma_{q}=\frac{1-(k+1) \exp \left(\frac{-2 \pi i(k) \alpha_{k+1}}{k+1}\right)+k \exp \left(-2 \pi i \alpha_{k+1}\right)}{k(k+1)\left[1-\exp \left(\frac{-2 q \pi i \alpha_{k+1}}{k+1}\right)\right]}
$$

Thus,

$$
\Lambda_{0}\left(\mathbf{B}_{k}, \mathbf{B}_{k+1}\right)=\frac{1-\exp \left(\frac{-2 \pi i k \alpha_{k+1}}{k+1}\right)}{k\left[1-\exp \left(\frac{-2 \pi i \alpha_{k+1}}{k+1}\right)\right]}
$$

Comparing (32) with $B_{k, 0}\left(\alpha_{k}\right)$, we can obtain:

$$
\alpha_{k+1}=\frac{k+1}{k} \alpha_{k}
$$

Finally, by substituting (22) into (33),

$$
\alpha_{k+1}=\frac{k+1}{4} \alpha_{4}
$$

We have completed the proof of Theorem 1.

According to Theorem 1 , if $\mathbf{W}_{m}^{\alpha_{m}}=\mathbf{W}_{n}^{\alpha_{n}}(m, n \geq 4)$, then

$$
\alpha_{m}=\frac{m}{n} \alpha_{n}
$$

Moreover, M-WFRFT can also satisfy the boundary axiom according to the following corollary.

Corollary 1 The boundary axiom and periodicity axiom for $m$-WFRFT $(m \geq 4)$ can be revealed as:

$$
\begin{aligned}
\mathbf{W}_{m}^{m / 4} & =\mathbf{F} \\
\mathbf{W}_{m}^{0} & =\mathbf{I} \\
\mathbf{W}_{m}^{\alpha_{m}+m} & =\mathbf{W}_{m}^{\alpha_{m}} .
\end{aligned}
$$

Proof According to [5], when $\alpha_{4}=1$, the following formula holds:

$$
\mathbf{W}_{4}^{1}=\mathbf{F}
$$

Moreover, $\alpha_{m}=\frac{m}{4} \alpha_{4}$ if $\mathbf{W}_{4}^{\alpha_{4}}=\mathbf{W}_{m}^{\alpha_{m}}$ via Theorem 1, then

$$
\mathbf{W}_{4}^{1}=\mathbf{W}_{m}^{\frac{m}{4}}=\mathbf{F}
$$

Similarly, (37) and (38) can be derived via Theorem 1.

The Corollary 1 can be straightforwardly derived via Theorem 1. Furthermore, Corollary 1 reveals a flexible method to switch between time domain and frequency domain with special modulation orders controlling.

Corollary 2 Let $\mathbf{G}_{4}$ and $\mathbf{G}_{m}$ be the $N \times N$ matrix spaces consisting of $\mathbf{W}_{4}^{\alpha_{4}}\left(\alpha_{4} \in \mathbf{R}\right)$ and $\mathbf{W}_{m}^{\alpha_{m}}\left(\alpha_{m} \in \mathbf{R}, m>4\right)$, that is, 


$$
\begin{aligned}
\mathbf{G}_{4} & =\left\{\mathbf{W}_{4}^{\alpha_{4}} \mid \alpha_{4} \in \mathbf{R}\right\} \\
\mathbf{G}_{m} & =\left\{\mathbf{W}_{m}^{\alpha_{m}} \mid \alpha_{m} \in \mathbf{R}\right\}
\end{aligned}
$$

Then, these two matrix spaces are equivalent.

Proof To any $\mathbf{W}_{4}^{\alpha_{4}} \in \mathbf{G}_{4}$, we find

$$
\mathbf{W}_{4}^{\alpha_{4}}=\mathbf{W}_{m}^{\frac{4}{m} \alpha_{m}}
$$

according to Theorem 1.

And,

$$
\mathbf{W}_{m}^{\frac{4}{m} \alpha_{m}} \in \mathbf{G}_{m}
$$

Thus,

$$
\mathbf{W}_{4}^{\alpha_{4}} \in \mathbf{G}_{m}
$$

That is to say, $\mathbf{G}_{4}$ is the subspace of $\mathbf{G}_{m}$. Similarly,

$$
\mathbf{W}_{m}^{\alpha_{m}}=\mathbf{W}_{4}^{\frac{m}{4} \alpha_{4}} \in \mathbf{G}_{4}
$$

holds for any $\mathbf{W}_{m}^{\alpha_{m}} \in \mathbf{G}_{m}$ via Theorem 1. Thus, $\mathbf{G}_{m}$ is also the subspace of $\mathbf{G}_{4}$.

In summary, the $\mathbf{G}_{4}$ space and $\mathbf{G}_{m}$ space are equivalent.

As a generalized of Corollary 2, $\mathbf{G}_{m}$ and $\mathbf{G}_{n}$ will be equivalent for any real $m, n$. This is obvious according to Corollary 2.

\section{Implementation structure of $2^{n}$-WFRFT and its complexity requirements}

As stated previously, we have proved the modulation order relationship between 4-WFRFT and $M$-WFRFT via Theorem 1. There would be a question about the next research in our mind. What is the significance to research the $M$-WFRFT since it can be represented by 4-WFRFT? As a special case of multi-WFRFT, we in this section will give the $2^{n}$-WFRFT $(n \geq 2)$ implementation structure and the complexity requirements.

\subsection{Implementation structure of $2^{n}$-WFRFT}

The $2^{n}$-WFRFT of the original signal $X$ with the length of $N$ can be expressed as:

$$
\mathfrak{F}^{\alpha} X=\mathbf{W}^{\alpha} X, n \geq 2 .
$$

Note that, we drop the subscript $2^{n}$ for brevity in this section. The $2^{n}$-WFRFT matrix, $\mathbf{W}^{\alpha}$, can be written as:

$$
\begin{aligned}
\mathbf{W}^{\alpha}= & \sum_{p=0}^{2^{n}-1} B_{p}(\alpha) \mathbf{W}_{4}^{\frac{4 p}{2^{n}}} \\
= & \sum_{p=0}^{2^{n-2}-1}\left[B_{p}(\alpha)+B_{p+2^{n-2}}(\alpha) \mathbf{F}\right. \\
& \left.+B_{p+2^{n-1}}(\alpha) \mathbf{P}+B_{p+3 \times 2^{n-2}}(\alpha) \mathbf{P F}\right] \mathbf{W}_{4}^{\frac{4 p}{2^{n}}},
\end{aligned}
$$

in which $\mathbf{P}$ is the permutation matrix with $[\mathbf{P}]_{m, n}=$ $\delta\left(\langle m+n\rangle_{N}\right)(m, n=0,1, \ldots, N-1)$, which can be as the inverse module. Due to the special structure of $2^{n}$. WFRFT, we can design the implementation structure, which can be the generalized form of that in [15]. We can implement the $2^{n}$-WFRFT using (46).

We provide the implementation structure of $2^{n}$-WFRFT and signal compositions in Fig. 1. Note that, when $n=$ 2 , the structure of $2^{n}$-WFRFT reduces to the well known structure of 4-WFRFT. The serial signal $X$ with length of $N$ was transmitted in parallel. According to (46), there are 4-WFRFT modules [15], discrete Fourier transform (DFT) modules, and inverse modules (termed as "P" in Fig. 1) in the implementation structure.

Different signals, dividing into different channels, will then be multiplied by the corresponding weight coefficients $\left(B_{j}, j=0, \ldots, 2^{n}-1\right)$. After taking the summation for all channels, the derived signal will be transmitted. And then, we obtain the output signal $Y$ by parallel and serial transform.

Note that there are two time domain signals over "channel 0 " and "channel $2^{n-1}$," which can be as the single carrier components. Moreover, there are two frequency domain

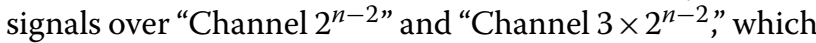
can be as the multi-carrier components $[1,3,15]$. Differing from [15], $2^{n}$-WFRFT involves $2^{n}-4$ weight fractional Fourier transform domain signals over the other channels, which can be as the hybrid carrier components $[1,3]$. These hybrid carrier components can be derived by different order 4-WFRFT transform. Therefore, $2^{n}$-WFRFT is a compatible modulation system of "single carrier," "multicarrier," and "hybrid carrier" modulations.

\subsection{Complexity requirements}

In this subsection, we will discuss the calculation complexity for $2^{n}$-WFRFT. The computation complexity consists of three parts: the 4-WFRFT modules, the DFT modules, and multiply modules. Computing a 4-WFRFT module costs $N \log N+4 N$ operations according to [3], where $N$ is the length of original signal. The calculation for each of DFT modules depends on FFT, which needs $N \log N$ operations. Another $2^{n} N$ multiplies will 


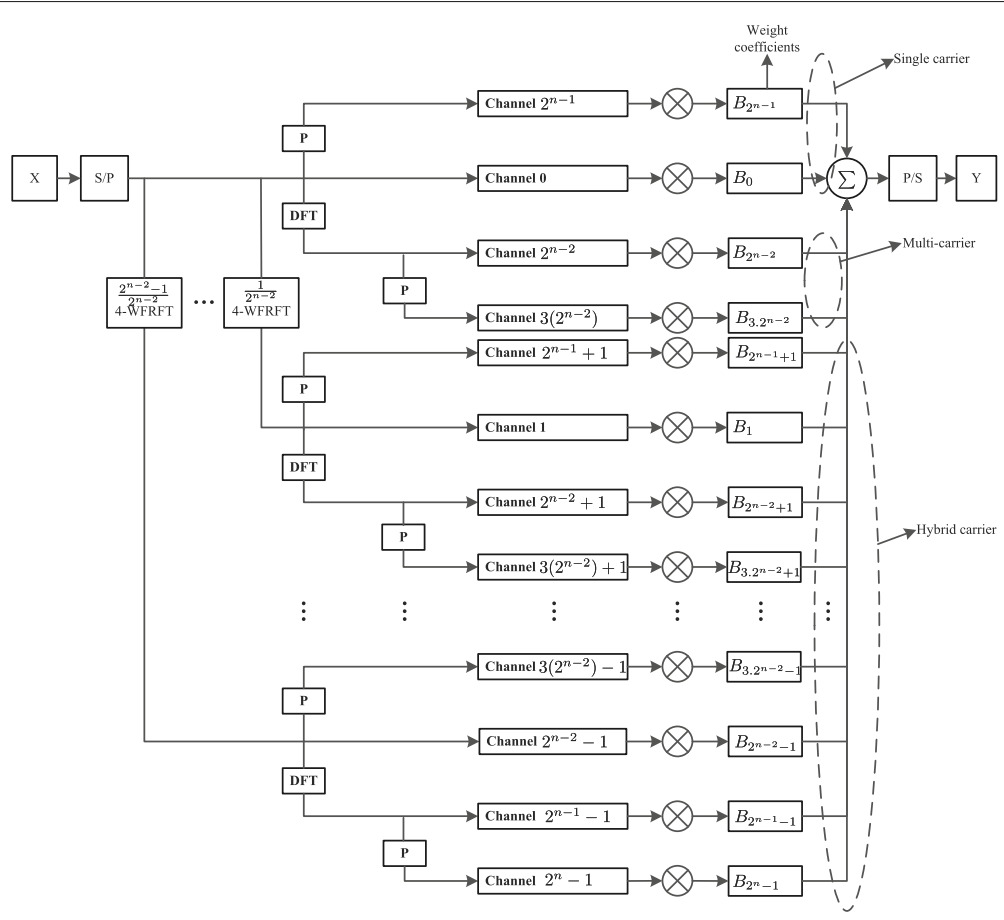

Fig. 1 Implementation structure of $2^{n}$-WFRFT and signal compositions

be employed in the weight coefficients multiply. The details of complexity requirements have been given in Table 1. Note that $n$ is the number of the channels (or antennas), in general, $n<N$. Besides, $2^{n}$ WFRFT will be degenerated into the classical 4-WFRFT for $n=2$.

\section{Simulation and potential applications analysis}

In order to verify the relationship between $\mathrm{m}$-WFRFT and n-WFRFT, we first provide a generalized hybrid carrier modulation system (GHCM) as shown in Fig. 2. The original signal $f$, assumed to be in the $\alpha$ order m-WFRFT domain, can be converted back to time domain through $\mathrm{a}-\alpha$ order $\mathrm{m}$-WFRFT at the transmitter. Generally, the received signal $f^{\prime}$, at the receiver, can be converted back to the original $\mathrm{m}$-WFRFT domain by a $\alpha$ order m-WFRFT. However, we can also obtain $f^{\prime}$, according to Corollary 1 , by a $\beta$ order $n$-WFRFT with $\beta=n / m \alpha$. This is a simple model without any equalization modular. Besides, the

Table 1 The details of complexity requirements

\begin{tabular}{lll}
\hline Operators & Complexity & Times \\
\hline 4-WFRFT & $\mathcal{O}(N \log N+4 N)$ & $2^{n-2}-1$ \\
DFT & $\mathcal{O}(N \log N)$ & $2^{n-2}$ \\
Multiply & $\mathcal{O}(N)$ & $2^{n}$ \\
\hline
\end{tabular}

GHCM system model, with $m=n=4$, can be degenerated to the classical hybrid carrier modulation system (HCM) $[1,3,6]$. The primary contribution of GHCM system is that the modulation orders (i.e., $\alpha$ and $\beta$ ) at the transmitter and receiver can be flexibly selected, which can expand the applications over communications (i.e., the security communication and MIMO systems). Finally, we also discuss the potential applications of multi-WFRFT over wireless communication.

\subsection{Simulation and analysis}

First, we will provide some simulations to verify the theorem on the relationship of modulation orders. Assuming the original signal is $x=\operatorname{sinc}(t)$, where $t \in$ $[-5,5]$. Here, we mainly want to verify the relationship between m-WFRFT and n-WFRFT. Therefore, the channel is assumed to be ideal without any interference and noise. The simulation results have been demonstrated from Figs. 3 and 4.

Figure 3 is the relationship between 8-WFRFT and 4WFRFT of the original signal. The original signal has been shown in Fig. 3a. Figure $3 \mathrm{~b}$ is the result of -0.8 order 8 WFRFT of original signal (here $m$ and $\alpha$ are 8 and 0.8 , respectively). The recover signal is derived by a 0.4 order 4-WFRFT. From Fig. 3c, we can clearly observe that the demodulation signal can be well coincide with the original signal. Moreover, the common communication signal 


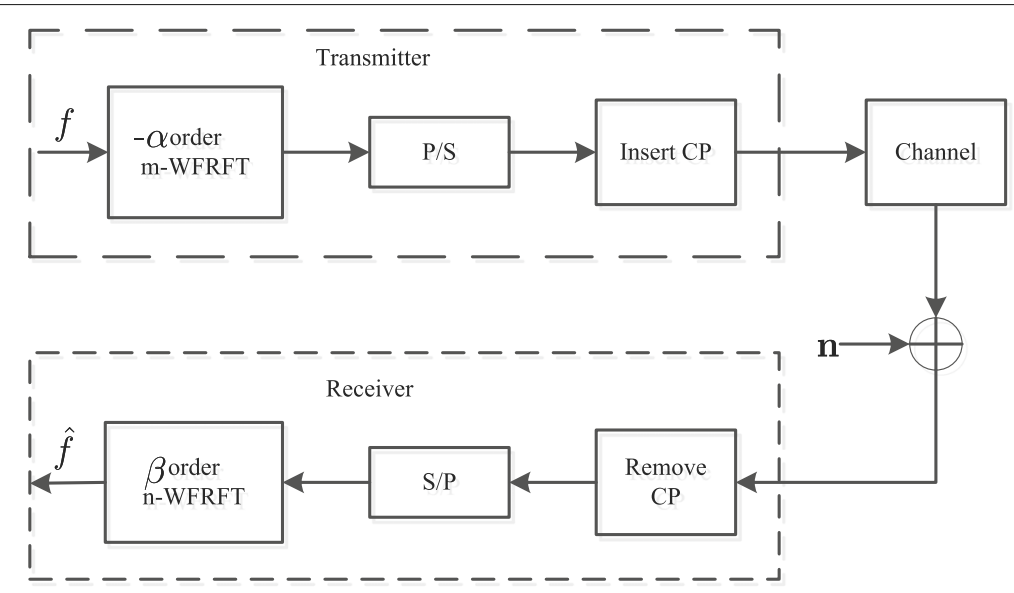

Fig. 2 The generalized hybrid carrier modulation (GHCM) system

to be simulated in Fig. 4a. Figure $4 \mathrm{~b}$ is the result of -0.6 order 16-WFFRFT of original signal (here $m$ and $\alpha$ are 16 and 0.6 , respectively). The recover signal is derived by a 0.3 order 8-WFRFT. From Fig. 4c, we can clearly observe that the demodulation signal can be well coincide with the original signal. Furthermore, according to the error between original signal and recover signal in Figs. 3d and $4 \mathrm{~d}$, we can also confirm the effectiveness of theorems above.

To verify the superiority of GHCM, we set the simulation under DS channels, in comparison with SCM and OFDM systems. We consider the 512 QPSK symbol for each block under DS channels. The bandwidth is $2 \mathrm{MHz}$. Moreover, the normalize Doppler frequency $f_{d} T_{d}=0.00384$, where $f_{d}$ is the single-side Doppler spread in $\mathrm{Hz}$ and $T_{d}$ denotes the sampling interval of the discrete-time system. The DS channel is modeled by a sixteen-tap WSSUS channel with an exponential multipath intensity profile [16]. In order to be fairly compared, we employ the partial FFT demodulation [1,3] in the three systems and the division number of 16 . Without loss of generality, we employ 8-WFRFT in the transmitter and 4-WFRFT in the receiver.

The simulation results have been shown in Fig. 5. The modulation order $\alpha$ of 1 , here $m=8$ and $n=4$ via Fig. 2, is selected in the transmitter. According to Theorem 1 , the demodulation order $\beta$, in the receiver, should be chose as 0.5. The selection of modulation order can be found in [17]. It is demonstrated that the superiority of the GHCM is obvious in comparison to OFDM systems under this DS channel. Moreover, GHCM performs better than SCM system when $E_{b} / N_{0} \geq 15 \mathrm{~dB}$. The GHCM system can be degenerated to HCM system [2] when $m=n=4$ via Fig. 2. However, the communication security performance of GHCM can be enhanced as the different order at the transmitter and receiver, in comparison to the HCM system. The reason will be provided in the next section.

\subsection{Potential applications of multi-WFRFT}

The communication security is a crucial problem to the wireless communication [18-21]. Two classes of methods for wireless communication security have been exploited. First, the transmitter and receiver have the different secret key and cannot be obtained by each other [21, 22]. Second, we also enhance the physical security in the wireless transmission [20]. In the multi-WFRFT scheme, we can design the communication system with the secrete key of the modulation order $\alpha$. According to Theorem 1, the transmitter and receiver may exploit different secrete keys to guarantee the wireless communication security. In this case, the order using at the transmitter is not necessary to be transmitted under wireless channels, it only transmits the encrypted signal and the order employing at the receiver. What is more, we can also employ the antenna array redundancy [19] to enhance the physical security due to the multi-antenna transmitting characteristics of multi-WFRFT. The advantage of the multi-WFRFT is that, it is not necessary to transmit the order of transmitter under wireless channels. Even if the illegitimate receiver intercepts the signal encrypted by $\mathrm{m}$-WFRFT at the transmitter, the signal cannot be correctly recovered without the order of $\mathrm{m}$-WFRFT. Thus, the theorem for multi-WFRFT is important for communication security.

The immediate application is to employ multi-WFRFT into Multi-Input Multi-Output (MIMO) system due to its multi-access sampling characteristics for multi-WFRFT, specially for $2^{n}$-WFRFT scheme. At the transmitter, we employ different antennas to transmit signals in the 
(a)

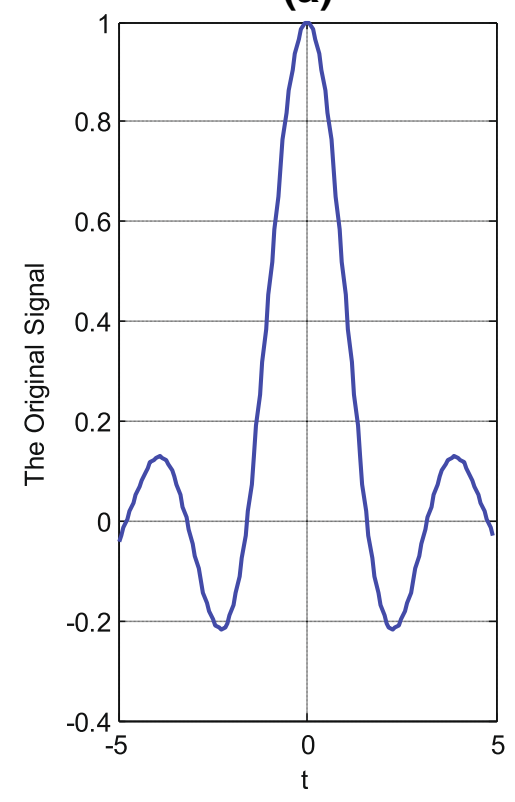

(c)

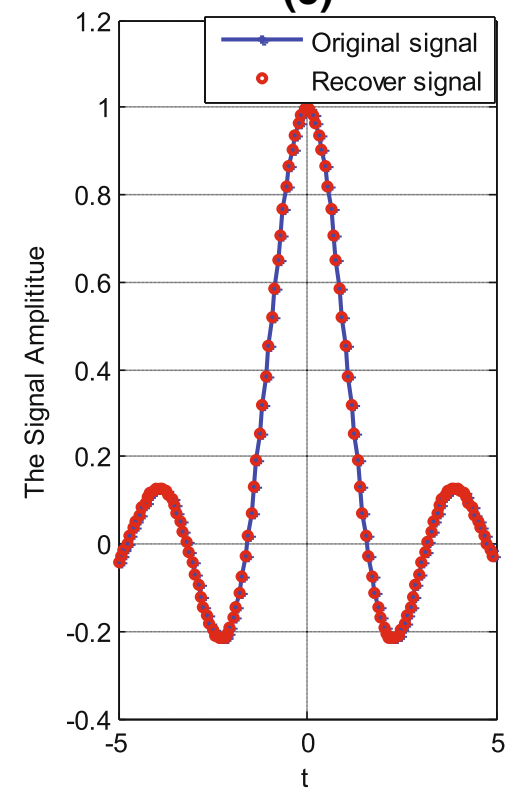

(b)

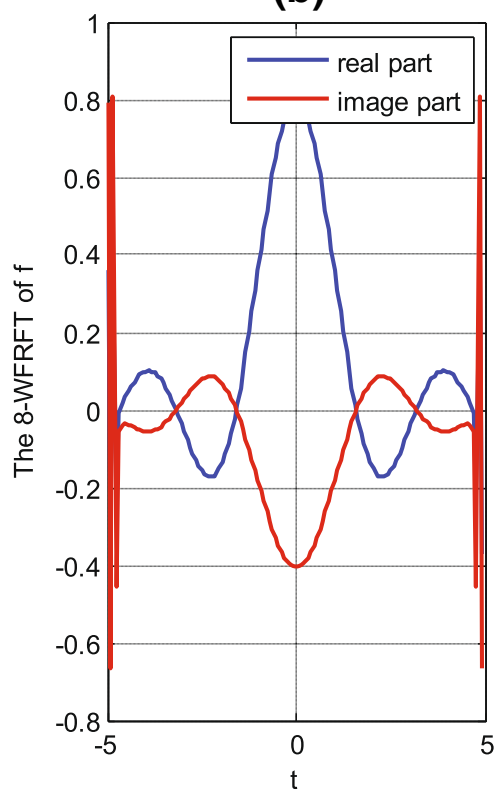

(d)

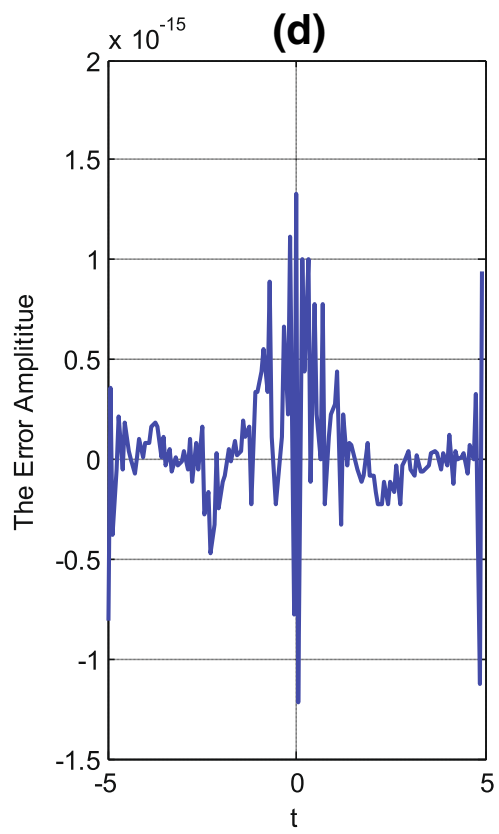

Fig. 3 The relationship between 8-WFRFT and 4-WFRFT of the original signal ( 8 -WFRFT signal. Moreover, —— is the original signal and o is the recover signal.) denotes the real part. And denotes the image part of

different channels of multi-WFRFT implement structure. We can receive the signals using multi-antennas in the receiver. It can take advantage of the channel diverse gain [23-25] of $2^{n}$-WFRFT scheme shown in Fig. 1.

4-WFRFT has various applications, i.e., channel equalization [1,3] and narrow-band interference (NBI) suppression [4], which can also be generalized into multi-
WFRFT scheme. Besides, the multi-WFRFT, as a new carrier convergence system, can also be exploited in the next generation cellular system.

\section{Conclusions}

In this paper, we focus on the modulation order relationship among different multi-WFRFT schemes and prove that $\alpha_{M}=(M / 4) \alpha_{4}$ if $\mathbf{W}_{M}^{\alpha_{m}}=\mathbf{W}_{4}^{\alpha_{4}}$. Moreover, we 
(a)

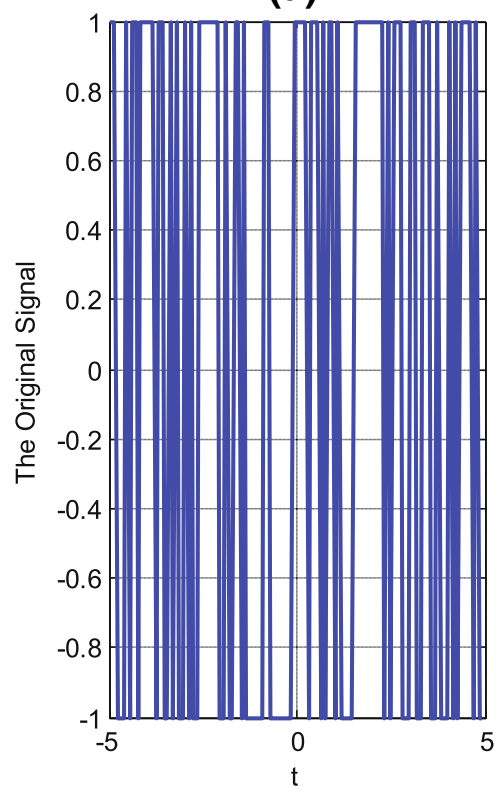

(c)

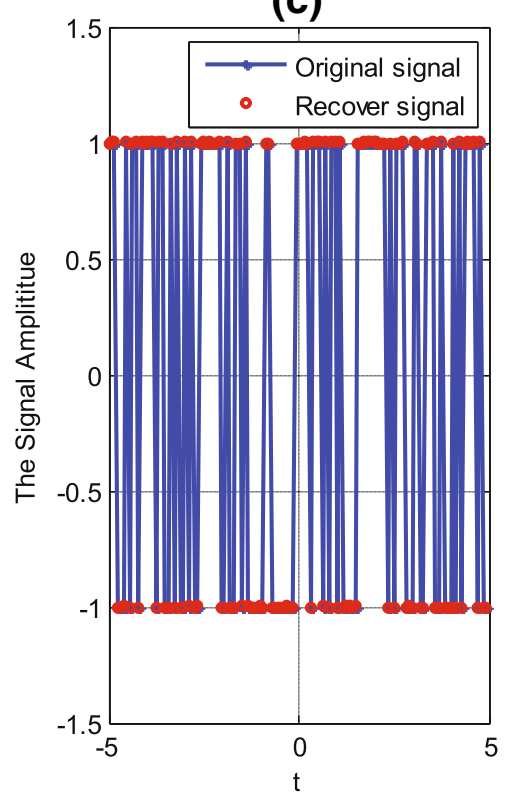

(b)

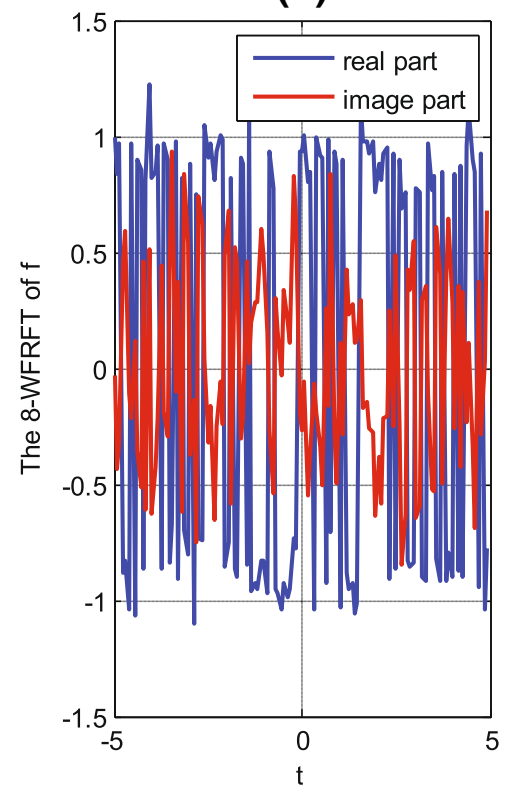

(d)

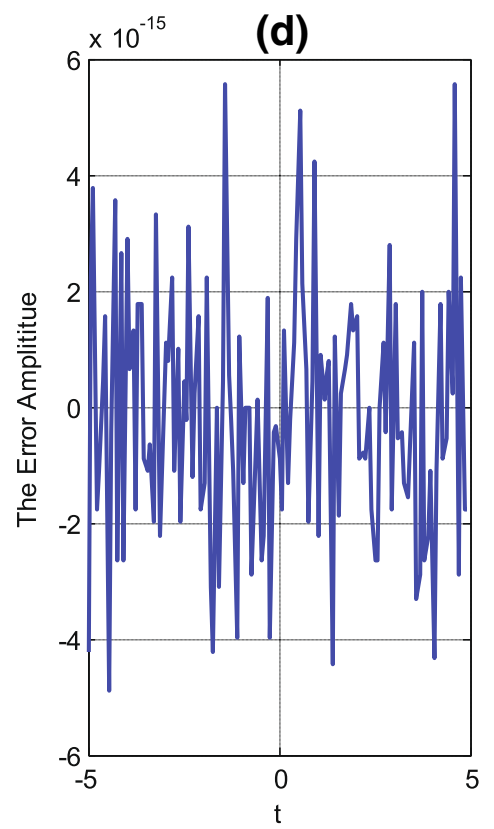

Fig. 4 The relationship between 16-WFRFT and 8-WFRFT of the original signal ( 16-WFRFT signal. Moreover, _— is the original signal and o is the recover signal.)

denotes the real part. And denotes the image part of

\footnotetext{
l.) denc
}

prove that the equality of 4-WFRFT space and multiWFRFT space through the corollary. In particular, the implementation structure for $2^{n}$-WFRFT, which is the convergence of single carrier, multi-carrier, and hybrid carrier components, has been well designed in this paper. According to the theorem of this paper, we propose a generalized hybrid carrier modulation (GHCM) system, which simplifies to the classical hybrid carrier modulation $(\mathrm{HCM})$ system with $m=n=4$. Also, the modulation or WFRFTs have numerical simu strated that, thr on multi-W and OFDM nels. Finally, multi-WFRFT MIMO system. 


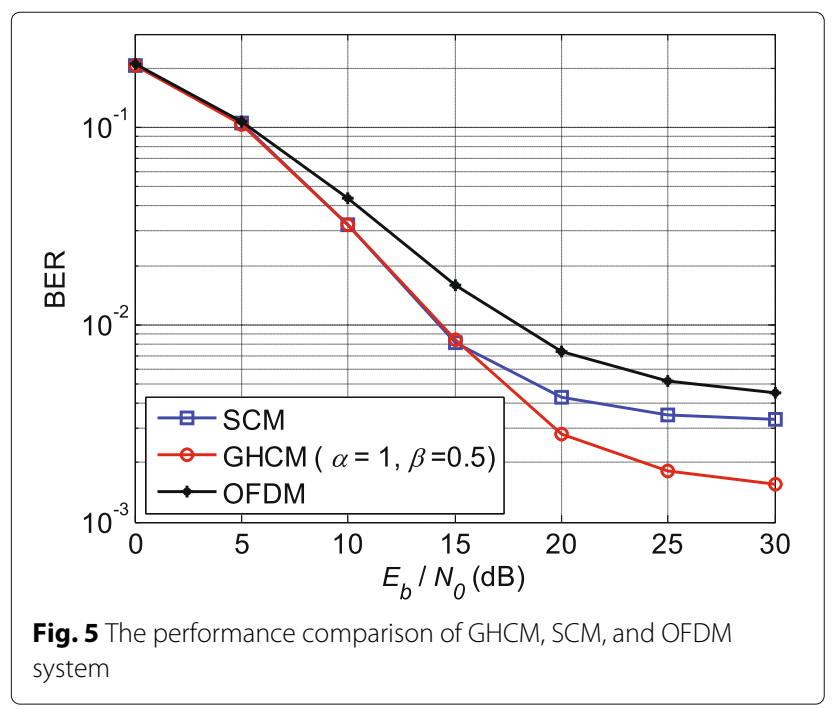

\section{Acknowledgements}

The authors would like to thank the editor and the anonymous referee for their valuable comments and suggestions that improved quality of this paper. This work is supported by the project funded by the China Postdoctoral Science Foundation under grant no. 2016M601286, the fund of Science and Technology on Communication Networks Laboratory under grant no. EX156410046, and the 973 Program under grant no. 2013CB329003. Moreover, this work is also supported by the fund of the National Key Laboratory of Science and Technology on Communications.

\section{Funding}

This work is supported by the project funded by the China Postdoctoral Science Foundation under grant no. 2016M601286, the fund of Science and Technology on Communication Networks Laboratory under grant no. EX156410046, and the 973 program under grant no. 2013 CB329003. Moreover, this work is also supported by the fund of the National Key Laboratory of Science and Technology on Communications.

\section{Availability of data and materials}

We assert that all of the data and materials in this paper are available, and all of the experiments can be reproduced according to this paper.

\section{Authors' contributions}

$Y L$ found the new theory and algorithm and wrote the whole paper as the main author. Prof. ZS and Prof. XS are the responsible for the revision of the whole paper and provided some useful proposal. All authors read and approved the final manuscript.

\section{Competing interests}

The authors declare that they have no competing interests.

\section{Publisher's Note}

Springer Nature remains neutral with regard to jurisdictional claims in published maps and institutional affiliations.

\section{Author details \\ 1 Science and Technology on Communication Networks Laboratory, Shijiazhuang, China. ${ }^{2}$ The 54th Research Institute of China Electronics Technology Group Corporation, Shijiazhuang, China. ${ }^{3}$ Communication Research Center, Harbin Institute of Technology, Harbin, China.}

\section{Received: 16 October 2017 Accepted: 5 February 2018}

Published online: 13 February 2018

\section{References}

1. Y Li, X Sha, K Wang, Hybrid carrier communication with partial FFT demodulation over underwater acoustic channels. IEEE Commun. Lett. 17(12), 2260-2263 (2013)
2. Y Li, X Sha, K Wang, Hybrid carrier modulation system with partial FFT demodulation over doubly selective channels in presence of carrier frequency offset. Circ. Syst. Signal Process. 33(12), 3967-3979 (2014)

3. Y Li, X Sha, FC Zheng, K Wang, Low complexity equalization of HCM systems with DPFFT demodulation over doubly-selective channels. IEEE Signal Process. Lett. 21(7), 862-865 (2014)

4. L Mei, Q Zhang, X Sha, N Zhang, WFRFT precoding for narrowband interference suppression in DFT-based block transmission systems. IEEE Commun. Lett. 17(10), 1916-1919 (2013)

5. Q Ran, DS Yeung, ECC Tsang, Q Wang, General multifractional fourier transform method based on the generalized permutation matrix group. IEEE Trans. Signal Process. 53(1), 83-98 (2005)

6. K Wang, X Sha, L Mei, X Qiu, Performance analysis of hybrid carrier system with MMSE equalization over doubly-dispersive channels. IEEE Commun. Lett. 16(7), 1048-1051 (2012)

7. G Cariolaro, T Erseghe, P Kraniauskas, N Laurenti, A unified framework for the fractional fourier transforms. IEEE Tran. Signal Process. 46(12), 3206-3219 (1998)

8. G Cariolaro, T Erseghe, P Kraniauskas, N Laurenti, Multiplicity of fractional fourier transform and their relationship. IEEE Tran. Signal Process. 48(1), 227-241 (2000)

9. T Erseghe, P Kraniauskas, G Cariolaro, Unified fractional fourier transform and sampling theorem. IEEE Tran. Signal Process. 47(12), 3419-3423 (1999)

10. HM Ozaktas, O Arikan, A Kutay, G Bozdagi, Digital computation of the fractional fourier transform. IEEE Tran. Signal Process. 44(9), 2141-2150 (1996)

11. SC Pei, MH Yeh, CC Tseng, Discrete fractional fourier transform based on orthogonal projections. IEEE Tran. Signal Process. 47(5), 1335-1348 (1999)

12. CC Shih, Fractionalization of fourier transform. Optics Commun. 118(1), 495-498 (1995)

13. JB Shah, MN Patel, Bayes estimation of shift point in geometric sequence. Commun. Stat. 36(6), 1139-1151 (2007)

14. AL Baker, Mathematical induction. Springer International Publishing. 30(2), 129-16 (2014)

15. L Mei, X Sha, N-T Zhang, The approach to carrier scheme convergence based on 4-weighted fractional fourier transform. IEEE Commun. Lett. 14(6), 503-505 (2010)

16. P Schniter, H Liu, in Proc.2003 Asilomar Conference on Signals, Systems and Computers. Iterative equalization for single-carrier cyclic-prefix in doubly-dispersive channels, vol. 1, (2003), pp. 502-506

17. CMa, X Sha, Y Hua, Hybrid carrier order selection scheme based on minimum mean square error equalization. IEEE Commun. Lett. 21(12) 2598-2601 (2017)

18. S Goel, R Negi, Guaranteeing secrecy using artificial noise. IEEE Trans. Wireless Commun. 7(6), 2180-2189 (2008)

19. X Li, J Hwu, EP Ratazzi, Using antenna array redundancy and channel diversity for secure wireless transmissions. J. Commun. 2(3), 24-32 (2007)

20. J Mo, M Tao, Y Liu, Relay placement for physical layer security: a secure connection perspective. IEEE Commun. Lett. 16(6), 878-881 (2012)

21. R Wilson, D Tse, RA Scholtz, Channel identification: secret sharing using reciprocity in ultrawideband channels. IEEE Trans. Inf. Forensics Secur. 2(3), 364-375 (2007)

22. X Fang, N Zhang, S Zhang, D Chen, X Sha, X Shen, On physical layer security: weighted fractional fourier transform based user cooperation. IEEE Trans. Wireless Commun. 16(8), 5498-5510 (2017)

23. N Dhahir, A Sayed, The finite-length multiple-input multiple-output MMSE-DFE. IEEE Tran. Signal Process. 48(10), 2921-2936 (2000)

24. M-W Kuan, C-W Kok, MMSE equalizer for MIMO-ISI channel with shorten guard period. IEEE Tran. Signal Process. 55(1), 389-395 (2007)

25. X Tang, Y Hua, Optimal design of non-regenerative MIMO wireless relays. IEEE Trans. Wireless Commun. 6(4), 1398-1407 (2007) 